ELECTRONIC RESEARCH ANNOUNCEMENTS OF THE AMERICAN MATHEMATICAL SOCIETY

Volume 4, Pages 12-17 (March 20, 1998)

S $1079-6762(98) 00041-9$

\title{
EIGENVALUE FORMULAS FOR THE UNIFORM TIMOSHENKO BEAM: THE FREE-FREE PROBLEM
}

\author{
BRUCE GEIST AND JOYCE R. MCLAUGHLIN
}

(Communicated by Michael Taylor)

\begin{abstract}
This announcement presents asymptotic formulas for the eigenvalues of a free-free uniform Timoshenko beam.
\end{abstract}

Suppose a structural beam is driven by a laterally oscillating sinusoidal force. As the frequency of this applied force is varied, the response varies. Experimental frequencies for which the response is maximized are called natural frequencies of the beam. Our goal is to address the question: if a beam's natural frequencies are known, what can be inferred about its bending stiffnesses or its mass density? To answer this question we need to know asymptotic formulas for the frequencies. Here we establish these formulas for a uniform beam.

One widely used mathematical model for describing the transverse vibration of beams was developed by Stephen Timoshenko in the 1920s (see [5], [6]). In this model, two coupled partial differential equations arise,

$$
\begin{gathered}
\left(E I \psi_{x}\right)_{x}+k A G\left(w_{x}-\psi\right)-\rho I \psi_{t t}=0, \\
\left(k A G\left(w_{x}-\psi\right)\right)_{x}-\rho A w_{t t}=P(x, t) .
\end{gathered}
$$

The dependent variable $w=w(x, t)$ represents the lateral displacement at time $t$ of a cross-section located $x$ units from one end of the beam. $\psi=\psi(x, t)$ is the cross-sectional rotation due to bending. $E$ is Young's modulus, i.e., the modulus of elasticity in tension and compression, and $G$ is the modulus of elasticity in shear. The nonuniform distribution of shear stress over a cross-section depends on cross-sectional shape. The coefficient $k$ is introduced to account for this geometry dependent distribution of shearing stress. $I$ and $A$ represent cross-sectional inertia and area, $\rho$ is the mass density of the beam per unit length, and $P(x, t)$ is an applied force. If we suppose the beam is anchored so that the so-called "free-free"

Received by the editors January 5, 1998.

1991 Mathematics Subject Classification. Primary 34Lxx; Secondary 73Dxx.

Key words and phrases. Timoshenko beam, asymptotic distribution of eigenvalues, boundary value problems.

The work of both authors was completed at Rensselaer Polytechnic Institute, and was partially supported by funding from the Office of Naval Research, grant number N00014-91J-1166. The work of the first author was also partially supported by the Department of Education fellowship grant number 6-28069. The work of the second author was also partially supported by the National Science Foundation, grant number DMS-9410700. 
boundary conditions hold (i.e., shearing forces and moments are assumed to be zero at each end of the beam), then $w$ and $\psi$ must satisfy the following four boundary conditions:

$$
w_{x}-\left.\psi\right|_{x=0, L}=0,\left.\quad \psi_{x}\right|_{x=0, L}=0 .
$$

After making a standard separation of variables argument, one finds that the Timoshenko differential equations for $w$ and $\psi$ lead to a coupled system of two second order ordinary differential equations for $y(x)$ and $\Psi(x)$,

$$
\begin{gathered}
\left(E I \Psi_{x}\right)_{x}+k A G\left(y_{x}-\Psi\right)+p^{2} I \rho \Psi=0, \\
\left(k A G\left(y_{x}-\Psi\right)\right)_{x}+p^{2} A \rho y=0 .
\end{gathered}
$$

Here, $p^{2}$ is an eigenvalue parameter. The conditions on $w$ and $\psi$ in (1) imply $y$ and $\Psi$ must satisfy the same free-free boundary conditions:

$$
y_{x}-\left.\Psi\right|_{x=0, L}=0,\left.\Psi_{x}\right|_{x=0, L}=0 .
$$

This boundary value problem for $y$ and $\Psi$ is self-adjoint, which implies that the values of $p^{2}$ for which nontrivial solutions to this problem exist, the eigenvalues for this model, are real. Furthermore, it is not difficult to show that the collection of all eigenvalues for this problem forms a discrete, countable, unbounded set of real nonnegative numbers. Moreover, it can be shown that if $\sigma$ is a natural frequency for a beam, then $p^{2}=(2 \pi \sigma)^{2}$ is one of the beam's eigenvalues. Therefore, it is possible to determine eigenvalues from natural frequency data obtained in an experiment like the one indicated in the opening paragraph.

Suppose from vibration experiments we have determined a set of natural frequencies for a beam with unknown elastic moduli and mass density, and have constructed a sequence of eigenvalues from this data. What information can the eigenvalues provide about these unknown material parameters? To address this question, we must determine how eigenvalues depend on $E, I, k G, A$ and $\rho$. This determination is not easy, since the dependence of eigenvalues on these coefficients is highly nonlinear. The first step towards general eigenvalue formulas for beams with nonconstant material and geometric parameters is to derive eigenvalue formulas for the uniform Timoshenko beam. (A beam is uniform when $E, k G, A, I$, and $\rho$ are constants.) In a forthcoming paper (and in Geist [1]), the formulas for the uniform free-free beam given below are crucial in establishing asymptotic formulas for the eigenvalues of free-ended beams which have variable density and constant material parameters otherwise.

This announcement presents asymptotic formulas for the eigenvalues of the freeended, uniform Timoshenko beam.

\section{A FREQUenCy EQUATION}

In this section a frequency equation is derived. The notation follows that given in Huang [3]. When $E, k G, A, I$, and $\rho$ are constant, the boundary value problem defined by (2), (3), and (4) may be written in the following simplified notation. Let $\xi=x / L, b^{2}=\rho A L^{4} p^{2} /(E I), r^{2}=I /\left(A L^{2}\right)$, and $s^{2}=E I /\left(k A G L^{2}\right)$. Interpreting a prime $\left({ }^{\prime}\right)$ as differentiation with respect to $\xi$, equations (2) and (3) can be proved equivalent to the differential equations

$$
s^{2} \Psi^{\prime \prime}-\left(1-b^{2} r^{2} s^{2}\right) \Psi+y^{\prime} / L=0,
$$


and

$$
y^{\prime \prime}+b^{2} s^{2} y-L \Psi^{\prime}=0
$$

similarly, the four boundary conditions given in (4) may be written as

$$
\left.\Psi^{\prime}\right|_{\xi=0,1}=0, \quad\left[\frac{y^{\prime}}{L}-\Psi\right]_{\xi=0,1}=0 .
$$

By eliminating $y$ or $\Psi$ from (5) or (6), we find that these two second order equations imply that $y$ and $\Psi$ must satisfy two decoupled fourth order ordinary differential equations

$$
\begin{gathered}
y^{(i v)}+b^{2}\left(r^{2}+s^{2}\right) y^{\prime \prime}-b^{2}\left(1-b^{2} r^{2} s^{2}\right) y=0 \\
\Psi^{(i v)}+b^{2}\left(r^{2}+s^{2}\right) \Psi^{\prime \prime}-b^{2}\left(1-b^{2} r^{2} s^{2}\right) \Psi=0 .
\end{gathered}
$$

Coupling between $y$ and $\Psi$ still occurs through the boundary conditions (7). Define $a$ and $B$ as

$$
\begin{aligned}
& a=\left[\frac{r^{2}+s^{2}}{2}-\sqrt{\left(\frac{r^{2}-s^{2}}{2}\right)^{2}+\frac{1}{b^{2}}}\right]^{1 / 2}, \\
& B=\left[\frac{r^{2}+s^{2}}{2}+\sqrt{\left(\frac{r^{2}-s^{2}}{2}\right)^{2}+\frac{1}{b^{2}}}\right]^{1 / 2} .
\end{aligned}
$$

In [3], Huang derives general solutions to equations (8) and (9), valid when $b^{2} r^{2} s^{2}$ is not 1 or 0 . These solutions are

$$
\begin{gathered}
y=c_{1} \cos b a \xi+c_{2} \sin b a \xi+c_{3} \cos b B \xi+c_{4} \sin b B \xi \\
\Psi=d_{1} \sin b a \xi+d_{2} \cos b a \xi+d_{3} \sin b B \xi+d_{4} \cos b B \xi
\end{gathered}
$$

The $c_{i}$ may be determined in terms of the $d_{i}$ by substituting the general solutions for $y$ and $\Psi$ into the second order differential equations (5) and (6); $y$ can then be expressed in terms of the $d_{i}$. For $b \neq 0$ or $1 / r s$, one can then show that solutions to the boundary value problem given in (5), (6), and (7) exist if and only if $\hat{A} \vec{d}=\overrightarrow{0}$, where $\vec{d}=\left(d_{1}, d_{2}, d_{3}, d_{4}\right)^{T}$ and

$$
\hat{A}=\left(\begin{array}{cccc}
0 & \frac{s^{2}}{a^{2}-s^{2}} & 0 & \frac{s^{2}}{-s^{2}+B^{2}} \\
b a & 0 & b B & 0 \\
\frac{s^{2} \sin b a}{a^{2}-s^{2}} & \frac{s^{2} \cos b a}{a^{2}-s^{2}} & \frac{s^{2} \sin b B}{B^{2}-s^{2}} & \frac{s^{2} \cos b B}{B^{2}-s^{2}} \\
b a \cos b a & -b a \sin b a & b B \cos b B & -b B \sin b B
\end{array}\right) .
$$

The matrix equation $\hat{A} \vec{d}=\overrightarrow{0}$ has nontrivial solutions if and only if the determinant of the matrix $\hat{A}$ vanishes. Let $\tilde{A}(b)=|\hat{A}|$. After carrying out some lengthy but routine calculations, it can be shown that

$$
\begin{aligned}
\tilde{A}(b)= & 2(1-\cos b a \cos b B) \\
& +\frac{b}{\left(b^{2} r^{2} s^{2}-1\right)^{1 / 2}} \cdot\left(b^{2} r^{2}\left(r^{2}-s^{2}\right)^{2}+3 r^{2}-s^{2}\right) \sin b a \sin b B .
\end{aligned}
$$


The matrix equation $\hat{A} \vec{d}=\overrightarrow{0}$ defines solutions to (5)-(7) whenever the (nonnegative) frequency parameter $b$ is not equal to 0 or $1 / r s .{ }^{1}$ This implies that when $b>0$ and $b \neq 1 / r s$, the free-free Timoshenko boundary value problem will have nontrivial solutions if and only if $\tilde{A}(b)=0$. Recall that the nonnegative eigenvalue parameter $p^{2}$ appearing in equations (2) and (3) satisfies $b=\sqrt{\rho A /(E I)} L^{2} p$. It follows that with the possible exception of $p=\left(r s L^{2}\right)^{-1} \sqrt{E I /(\rho A)}, p>0$ is the square root of a nonzero eigenvalue if and only if $\tilde{A}\left(\sqrt{\rho A /(E I)} L^{2} p\right)=0$. The eigenvalue formulas documented in the next section are established by estimating the roots of the frequency function $\tilde{A}$.

\section{Asymptotic formulas}

The asymptotic formulas for the eigenvalues of the free-free uniform Timoshenko beam are presented in the theorem below. The discussion following the theorem is intended to provide an overview of the theorem's proof. For a comprehensive and thorough demonstration of the following result, the interested reader should consult [1, pp. 54-128].

Theorem 1. Let $E, k G, I, A$, and $\rho$ all be positive constants with $E \neq k G$, and let

$$
0<\bar{p}_{1} \leq \bar{p}_{2} \leq \cdots \leq \bar{p}_{i} \leq \bar{p}_{i+1} \leq \cdots
$$

be the positive square roots of eigenvalues for the uniform Timoshenko beam with free ends. Let

$$
0<p_{1} \leq p_{2} \leq \cdots \leq p_{i} \leq p_{i+1} \leq \cdots
$$

be the positive roots of the function

$$
\sin \left(\frac{L \rho^{1 / 2}}{\sqrt{E}} p\right) \sin \left(\frac{L \rho^{1 / 2}}{\sqrt{k G}} p\right) .
$$

Then there exist fixed integers $\hat{I}_{1}$ and $\tilde{N}$ such that if $i>\hat{I}_{1}$, then

$$
\left|p_{i}-\bar{p}_{i+\tilde{N}}\right|<O\left(1 / p_{i}\right) .
$$

Now suppose $\left\{p_{i_{n}}\right\}_{n=1}^{\infty} \subset\left\{p_{i}\right\}_{i=1}^{\infty}$ such that for some fixed but arbitrary $e \in(0,1)$,

$$
\sin \left(\frac{L \rho^{1 / 2}}{\sqrt{E}} p_{i_{n}}\right)=0
$$

and

$$
\left|\sin \left(\frac{L \rho^{1 / 2}}{\sqrt{k G}} p_{i_{n}}\right)\right|>e, \quad n=1,2, \ldots
$$

\footnotetext{
${ }^{1}$ For a discussion of when $b=1 / r s$ defines an eigenvalue, see [1, pp. 26-29]. For any choice of the beam parameters $E, k G, \rho, I$ and $A$, the equation $b=0$ defines an eigenvalue. In this case, if $k_{1}$ and $k_{2}$ are arbitrary constants, then $\left(\begin{array}{c}Y \\ \Psi\end{array}\right)=k_{1}\left(\begin{array}{c}L \xi \\ 1\end{array}\right)+k_{2}\left(\begin{array}{c}0 \\ 1\end{array}\right)$ solves the boundary value problem (5)-(7). Since the two vectors on the right of the last equation are linearly independent, two linearly independent solutions to (5)-(7) always exist when $b=0$. Hence $b=0$ always defines the "double" eigenvalue $p^{2}=0$ for the free-free Timoshenko beam. Solutions to the boundary value problem when $b=0$ correspond to rigid body rotation and displacement of the beam. Nonzero double eigenvalues are also possible. See Geist and McLaughlin [2] for a discussion and examples of nonzero double eigenvalues.
} 
Then there is an $\hat{I}_{2}$ such that $i_{n}>\hat{I}_{2}$ implies

$$
\bar{p}_{i_{n}+\tilde{N}}^{2}=p_{i_{n}}^{2}+\left(1-\frac{1}{2} \frac{k G+E}{k G-E}\right) \frac{A}{I} \frac{k G}{\rho}+O\left(1 / p_{i_{n}}\right) .
$$

Similarly, if $\left\{p_{j_{m}}\right\}_{m=1}^{\infty} \subset\left\{p_{i}\right\}_{i=1}^{\infty}$ is such that

$$
\sin \left(\frac{L \rho^{1 / 2}}{\sqrt{k G}} p_{j_{m}}\right)=0
$$

and

$$
\left|\sin \left(\frac{L \rho^{1 / 2}}{\sqrt{E}} p_{j_{m}}\right)\right|>e, \quad m=1,2, \ldots,
$$

then there is an $\hat{I}_{3}$ such that $j_{m}>\hat{I}_{3}$ implies

$$
\bar{p}_{j_{m}+\tilde{N}}^{2}=p_{j_{m}}^{2}+\left(1+\frac{1}{2} \frac{k G+E}{k G-E}\right) \frac{A}{I} \frac{k G}{\rho}+O\left(1 / p_{j_{m}}\right) .
$$

\section{Proof Sketch}

Suppose $f$ and $g$ are defined as

$$
\begin{aligned}
& f=\left(\frac{3 r^{2}-s^{2}}{b^{2}}+r^{2}\left(r^{2}-s^{2}\right)^{2}\right) \sin b a \sin b B \\
& g=\frac{2(1-\cos b a \cos b B) \sqrt{r^{2} s^{2}-\frac{1}{b^{2}}}}{b^{2}}
\end{aligned}
$$

then $\tilde{A}(b)$ can be written as

$$
\tilde{A}(b)=\frac{b^{2}}{\sqrt{r^{2} s^{2}-\frac{1}{b^{2}}}}(f+g) .
$$

For $b>0, \tilde{A}(b)=0$ if and only if $f+g=0$. It is instructive to observe that for $b>1 / r s$, the arguments $b a$ and $b B$ of the trigonometric functions appearing in the expressions defining $f$ and $g$ are strictly increasing in $b$. More importantly, as $b$ increases, $g$ converges to zero and $f$ converges to the function $r^{2}\left(r^{2}-s^{2}\right)^{2} \sin b a \sin b B$. It follows then that for large $b$, the roots of $\tilde{A}$ are approximated by the roots of $\sin b a \sin b B$. In [1] it is shown that the isolated roots of the function $\sin b a \sin b B$, that is, those roots of $\sin b a \sin b B$ that are separated from neighboring roots of $\sin b a \sin b B$ by more than some arbitrarily chosen fixed distance, occur along the $b$-axis at most an $O\left(1 / b^{2}\right)$ distance from the nearest roots of $\tilde{A}$. Furthermore, it is shown in [1] that every root of $\sin b a \sin b B$, regardless of whether or not it is well separated from the neighboring roots of $\sin b a \sin b B$, occurs no further than an $O(1 / b)$ distance from the nearest zero of the function. These results prove that near every root of $\sin b a \sin b B$, at least one root of $\tilde{A}$ lies nearby.

In [1] it is also shown (using Rouché's Theorem in the complex plane) that there exists an unbounded, strictly increasing infinite sequence $\sigma_{j}, j=1,2, \ldots$, which defines a sequence of intervals $\left[\sigma^{*}, \sigma_{j}\right]$ in which the functions $\tilde{A}$ and $\sin b a \sin b B$ have exactly the same number of zeros. Hence for $b \in\left[\sigma^{*}, \sigma_{j}\right], j=1,2, \ldots$, there is a one-to-one correspondence between the roots of $\sin b a \sin b B$ and the roots of $\tilde{A}(b)$. For $b$ large enough, the roots of $\sin b a \sin b B$ can be matched up one-to-one with the nearest roots of $\tilde{A}(b)$ in such a way that each root of $\sin b a \sin b B$ lies no further 
than an $O(1 / b)$ distance away from the corresponding nearest root of $\tilde{A}$. For those roots of $\tilde{A}$ near isolated roots of $\sin b a \sin b B$, the distance between such roots of $\tilde{A}$ and the corresponding nearest roots of $\sin b a \sin b B$ is $O\left(1 / b^{2}\right)$. Even though $a$ and $B$ defined in (10) and (11) are nonlinear functions of the frequency parameter $b$, it is possible to explicitly determine all roots of the function $\sin b a \sin b B$. Eigenvalues satisfying conditions (15) and (16) or conditions (18) and (19) correspond to roots of the frequency function that are near isolated roots of $\sin b a \sin b B$. For all such eigenvalues, formulas for the roots of $\sin b a \sin b B$ are accurate enough approximations to the roots of $\tilde{A}$ that they can be used to derive the estimates given in (17) and (20).

To see why the $O\left(1 / b^{2}\right)$ bound applies to isolated roots of $\sin b a \sin b B$, let $b_{0}$ be such an isolated root; then $b_{0}$ is an isolated simple root of $f$. When $b$ is near $b_{0}$, $d f / d b$ must be bounded away from zero. Since for all $b>1 / r s, 0 \leq g(b)<2 r s / b^{2}$, it follows that if $b_{0}$ is large enough, then $f\left(b_{0}+\Delta b\right)=-g\left(b_{0}+\Delta b\right)$ for some $\Delta b=O\left(1 / b_{0}^{2}\right)$, and hence that $\tilde{A}\left(b_{0}+O\left(1 / b_{0}^{2}\right)\right)=0$. To gain some insight into why only an $O(1 / b)$ bound (and not necessarily an $O\left(1 / b^{2}\right)$ bound) applies to roots of $\tilde{A}$ near nonisolated roots of $\sin b a \sin b B$, suppose $n$ and $m$ are positive integers such that $n-m$ is odd, and that $b_{1}$ satisfies $b_{1} a\left(b_{1}\right)=n \pi$ and that $b_{2}$ satisfies $b_{2} B\left(b_{2}\right)=m \pi$, where $b_{1}$ and $b_{2}$ are very near one another. (Nonisolated roots $b_{1}$ and $b_{2}$ of $\sin b a \sin b B$ satisfying these requirements exist for many choices of the beam parameters. Existence of double roots is shown in Geist [1]. See also Geist and McLaughlin [2].) For values of $b$ near $b_{1}$ and $b_{2}, g(b) \approx 2 r s / b^{2}$, and $d f /\left.d b\right|_{b_{i}}, i=1,2$, is small (since $b_{1}$ and $b_{2}$ must be located near one of the local minima of $f$ ). Hence, the dominant term in a Taylor series expansion for $f$ about $b_{i}, i=1$ or 2 , will be quadratic in $b-b_{i}=\Delta b$ when $b$ is small. This implies the equation $f\left(b_{i}+\Delta b\right)=-g\left(b_{i}+\Delta b\right) \approx-2 r s /\left(b_{i}+\Delta b\right)^{2}$ will have a solution when $\Delta b \approx\left[O\left(1 / b_{i}^{2}\right)\right]^{1 / 2}=O\left(1 / b_{i}\right)$, which implies that $\tilde{A}\left(b_{i}+O\left(1 / b_{i}\right)\right)=0$.

\section{REFERENCES}

[1] Bruce Geist, The asymptotic expansion of the eigenvalues of the Timoshenko beam, Ph.D. Dissertation, Rensselaer Polytechnic Institute, Troy, NY, 1994.

[2] Bruce Geist and J. R. McLaughlin, Double eigenvalues for the uniform Timoshenko beam, Applied Mathematics Letters, 10 (1997), 129-134. CMP 97:15

[3] T. C. Huang, The effect of rotatory inertia and of shear deformation on the frequency and normal mode equations of uniform beams with simple end conditions, Journal of Applied Mechanics 28 (1961), 579-584. MR 24:B579

[4] Edwin T. Kruszewski, Effect of transverse shear and rotary inertia on the natural frequency of a uniform beam, National Advisory Committee for Aeronautics, Technical Note no. 1909, July 1949.

[5] Stephen Timoshenko, On the correction for shear of the differential equation for transverse vibrations of prismatic bars, Philisophical Magazine 41 (1921), 744-746.

[6] - On the transverse vibrations of bars of uniform cross-section, Philisophical Magazine 43 (1922), 125-131.

[7] R. W. Trail-Nash and A. R. Collar, The effects of shear flexibility and rotatory inertia on the bending vibrations of beams, Quart. J. Mech. Appl. Math. 6 (1953), 186-222. MR 16:541f

Unisys Corporation, 41100 Plymouth Road, Plymouth, Mi 48170

E-mail address: Bruce.Geist@unisys.com

Department of Mathematical Sciences, Rensselaer Polytechnic Institute, Troy, Ny 12180

E-mail address: mclauj@rpi.edu 